\title{
REDES SOCIAIS E COOPERAÇÃO: UM ESTUDO BIBLIOMÉTRICO
}

\section{SOCIAL NETWORKS AND COOPERATION: A BIBLIOMETRIC STUDY}

\author{
Ana Paula Lopes* E-mail: aplopes10@hotmail.com \\ Marly Monteiro de Carvalho*E-mail: marlymc@usp.br \\ Afonso Carlos Corrêa Fleury* E-mail: acfleury@usp.br \\ ** Universidade de São Paulo (USP), São Paulo, SP
}

\begin{abstract}
Resumo: A análise de redes sociais envolve ciência social e comportamental. $O$ processo de descentralização das atividades produtivas, como a formação de "empresas-rede", resultado do enxugamento das grandes estruturas corporativas de outrora, marcado pela subcontratação (outsoucing) e formação de alianças, mostra a importância desse tema. O objetivo principal deste artigo é analisar a teoria de cooperação e redes sociais, num período de 24 anos. Para isso, foi realizado um estudo bibliométrico com análise de conteúdo. A base de dados escolhida para a busca da amostra inicial foi a ISI Web of Science. Os tópicos de busca foram "social network" e "cooperation". Foram analisados 97 artigos e suas referências, por meio de redes de citações. Os principais grupos de pesquisa identificados tratam de temas relacionados com confiança, alianças estratégicas, cooperação natural, teoria dos jogos, capital social, intensidade da interação, reciprocidade e inovação. Verificou-se que as publicações ocorreram em um grande número de periódicos, o que indica que o tema é multidisciplinar, sendo que apenas cinco periódicos publicaram ao menos três artigos. Apesar de a primeira publicação datar de 1987, foi a partir de 2006 que as publicações efetivamente aumentaram. As áreas mais relacionadas com a temática da pesquisa foram desempenho, evolução, gestão, gráficos, modelo e teoria dos jogos.
\end{abstract}

Palavras-chave: Redes sociais. Cooperação. Colaboração.

Abstract: The social network analysis involves social and behavioral science. The decentralization of productive activities, such as the formation of "network organizations" as a result of downsizing of large corporate structures of the past, marked by outsoucing and formation of alliances, shows the importance of this theme. The main objective of this paper is to analyze the theory of cooperation and social networks over a period of 24 years. For this, was performed a bibliometric study with content analysis. The database chosen for the initial sample search was ISI Web of Science. The search topics were "social network" and "cooperation". Were analyzed 97 articles and their references, through networks of citations. The main identified research groups dealing with issues related to trust, strategic alliances, natural cooperation, game theory, social capital, intensity of interaction, reciprocity and innovation. It was found that the publications occurred in a large number of journals, which indicates that the theme is multidisciplinary, and only five journals published at least three articles. Although the first publication has occurred in 1987, was from 2006 that the publications effectively increased. The areas most related to the theme of the research were performance, evolution, management, graphics, model and game theory.

Keywords: Social networks. Cooperation. Collaboration.

\section{INTRODUÇÃO}

Apesar de não ser recente, a literatura de redes sociais modificou-se e tornouRevista Produção Online, Florianópolis, SC, v.13, n. 2, p. 634-654, abr./jun. 2013. 
se relevante nos últimos anos (WASSERMAN; FAUST, 1994), sob o alicerce dos conceitos de custo de transação e capital social (WILLIAMSON, 1992; WALKER et al., 1997). Após um período de predomínio da produção em massa, surgiu a necessidade de uma forma flexível e descentralizada de produção, que criou o conceito de "empresa rede", envolvendo relações intra e inter empresas (SCHMITZ, 1989; CHESNAIS, 1996; CANDIDO; VIEIRA, 2006). As grandes estruturas corporativas de outrora passaram a dividir espaço com entidades organizadas por meio de distintas formas de interação, tais como alianças estratégicas, parcerias e joint ventures. O capital social ganhou destaque, influenciando diretamente a formação, desenvolvimento e sobrevivência das então chamadas redes sociais (OLK; ELVIRA, 2001; CHAVERRI, 1997; FLAP; VOLKER, 2001).

Pesquisas anteriores consideraram redes sociais um meio de transferência rápido e simples de informação e de conhecimento, com o objetivo principal de atingir metas e obter vantagens competitivas (CHWE, 1999; RIVELLINI et al., 2006; FOWLER et al., 2009). Além de compreender os mecanismos de modelagem destas redes (IACOBUCCI; HOPKINS, 1992), tornou-se necessário conhecer a intensidade das relações de interação (HU et al., 2007, 2008), a dinâmica destas redes (DU et al., 2002), os fatores motivadores de entrada, o conceito de coopetição, a existência ou não de propensão à inovação (CANTNER; GRAF, 2006; GIULIANI; BELL, 2005) e a confiança (FERRIN et al., 2006).

Tem crescido, também na comunidade acadêmica nacional, o interesse por estudos focados em relações de cooperação, bem como por estudos bibliométricos (LOPES e CARVALHO, 2012; TALAMO e CARVALHO, 2010a; TALAMO e CARVALHO, 2010b; OPRIME et al., 2010).

Neste contexto, o objetivo deste trabalho é analisar a teoria de redes sociais e cooperação, com o intuito de identificar autores e trabalhos relevantes, periódicos que mais publicaram, áreas temáticas representativas, bem como possíveis grupos de pesquisa. $O$ trabalho pretende responder a questão de como a literatura de redes sociais e cooperação evoluiu entre os anos de 1997 e 2010. Para cumprir seus objetivos, o trabalho primeiramente estabelece o referencial teórico da pesquisa, seguido pelos métodos e técnicas adotados, resultados alcançados e, finalmente, suas conclusões. 


\section{REDES SOCIAIS E COOPERAÇÃO: SÍNTESE DO QUADRO TEÓRICO}

Dada a complexidade e velocidade das inovações, cada vez mais é preciso estar conectado em rede (CARVALHO, 2009; AMATO NETO, 2000; CARDOSO; CARDOSO; CASAROTTO FILHO, 2011). Walker et al. (1997) verificaram que para entender as relações de cooperação é preciso analisar a rede como um todo. Kemczinski et al. (2007) Identificaram que, apesar de alguns autores considerarem cooperação como sinônimo de colaboração, os dois termos diferem entre si e são complementares.

Segundo Harabi (2002), as principais vantagens da cooperação entre concorrentes são: superar as limitações financeiras de Pesquisa \& Desenvolvimento; obter ganhos de escala em Pesquisa \& Desenvolvimento; internacionalização de spillovers tecnológicos; maior sinergia uma vez que cada empresa contribui com o que tem de melhor em termos de "capability"; redução de riscos e incertezas.

Alguns autores acreditam que a primeira pessoa a ter utilizado o termo "redes sociais" tenha sido o autor Barnes, em seu trabalho de 1954, no qual estudou padrões de interação entre pessoas (WASSERMAN; FAUST, 1994).

Trata-se de uma estrutura endógena, a qual depende das escolhas individuais dos seus atores (EGUILUZ, 2005), cuja formação consiste basicamente de nós e vínculos, onde os nós são os seus integrantes (atores) e os seus vínculos representam a relação de interação entre os mesmos (MCDONALD, 2007).

Segundo Flap e Volker (2001), as redes são uma forma de atingir metas, as quais não poderiam ser alcançadas de maneira distinta e, o seu número de integrantes, bem como a habilidade dos mesmos em cooperar, representam o seu capital social. O desenvolvimento do capital social influencia diretamente não só a formação da rede, mas também o crescimento da indústria (WALKER et al., 1997). O Anexo 1 mostra uma tipologia de redes, segundo o trabalho de Wasserman e Faust (1994).

A análise de redes sociais pode ser definida como sendo uma metodologia de análise interdisciplinar desenvolvida por sociólogos e pesquisadores na área de psicologia social, em parceria com matemáticos e estatísticos (CANTNER; GRAF, 2006). Sua teoria provê métricas quantitativas para análises complexas de interações.

Revista Produção Online, Florianópolis, SC, v.13, n. 2, p. 634-654, abr./jun. 2013. 
Estudos e pesquisas relativos à constituição de redes de cooperação produtiva destacam-se como temas pertinentes à engenharia de produção (AMATO NETO, 2006), demonstrando a necessidade de novos arranjos interorganizacionais. Cada vez mais o padrão de vantagem competitiva é cooperativo, com a atuação de diversos atores, como por exemplo, empresas, universidades e até comunidades de consumidores.

\section{MÉTODO DE PESQUISA}

O método de pesquisa utilizado foi o estudo bibliométrico com análise de conteúdo. Bibliometria pode ser definida como uma série de técnicas que visam quantificar o processo da comunicação escrita (IKPAAHINDI, 1985). Uma área relevante da bibliometria é a análise de citações, que é a parte da bibliometria que investiga as relações entre os documentos citantes e os documentos citados considerados como unidades de análise, no todo ou em suas diversas partes: autor, título, origem geográfica, ano, idioma de publicação, dentre outros (FORESTI, 1989; NEELY, 2005). Para Prasad e Tata (2005), o estudo bibliométrico permite identificar padrões na literatura, analisando periódicos e áreas de destaque.

\subsection{Amostra}

A amostra inicial de trabalhos foi obtida por meio da utilização das palavras chaves "social network" e "cooperation", na base de dados ISI Web of Science, resultando em 161 trabalhos. Foi utilizada como filtro a seleção apenas de artigos, diminuindo para 101 o número de trabalhos. Optou-se por analisar apenas artigos pelo fato deles conterem os meta dados necessários para a análise bibliométrica, tais como autores, periódicos, número de citações, referências bibliográficas, entre outras. Destes 101 trabalhos, 4 foram desconsiderados por não se adequarem ao tema, resultando numa amostra de 97 artigos.

O período analisado foi de 1987 a 2010. A Figura 1 apresenta o fluxo de trabalho dos artigos. 
Figura 1 - Fluxo de trabalho dos artigos.

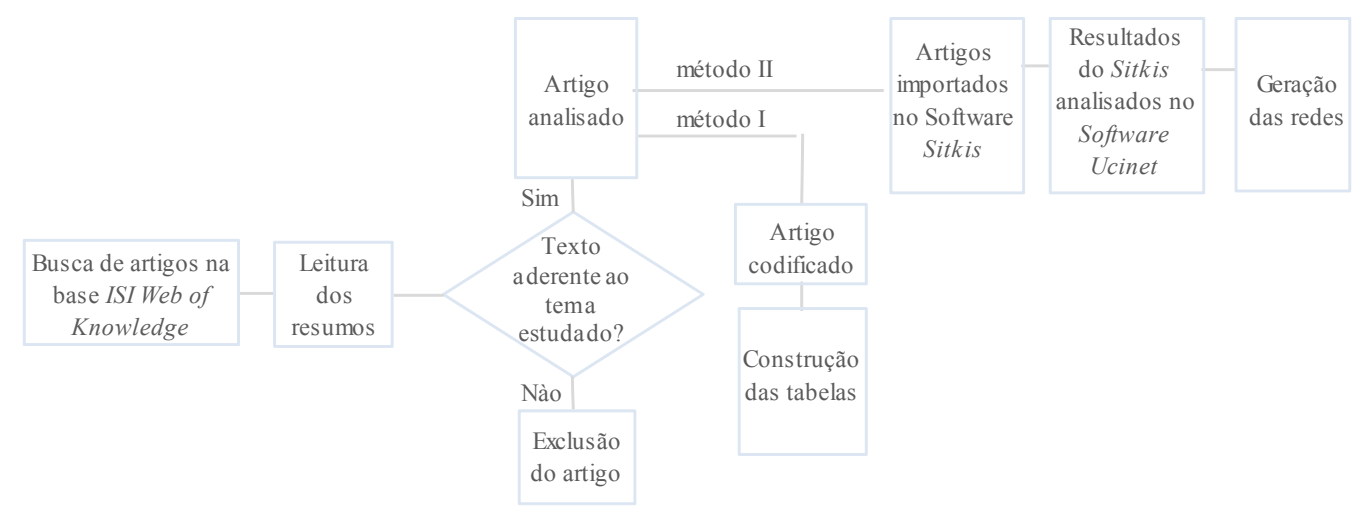

\section{RESULTADOS}

As seções seguintes apresentam os principais resultados da pesquisa.

\subsection{Periódicos que mais publicaram}

O Anexo 2 mostra o número de publicações por ano.

Os periódicos que tiveram maior número de publicações foram:

- Behavioral Ecology and Sociobiology: suas publicações são voltadas para estudos quantitativos empíricos e teóricos na análise de interações entre animais. Possui um fator de impacto de 2.917.

- Organization Science: com um fator de impacto de 3.130, abrange áreas como estratégia, gestão e teoria organizacional.

- Physica A-Statistical Mechanics and its Applications: reconhecido pela sociedade europeia de física, suas publicações abrangem o campo da mecânica estatística. Tem um fator de impacto de 1.441 e publica uma média de 24 edições por ano.

- Proceedings of the National Academy of Sciences of the USA: voltado para as ciências físicas, biológicas e sociais, possui um fator de impacto de 9.643.

- Research Policy: trata-se de um periódico multidisciplinar, que estuda áreas como inovação, pesquisa \& desenvolvimento, tecnologia e ciência. Tem uma média de 10 edições por ano e um fator de impacto de 2.655 . 
Para a construção das redes foram utilizados dois software: Sitkis 2.0 (Schildt, 2002) e Ucinet for Windows - Version 6.289 (Borgatti et al, 2002). A Figura 2 mostra a rede de palavras chaves que apareceram mais do que sei vezes, na amostra de 97 artigos. O filtro para o mínimo de citações foi feito com base no manual do software "Sitkis", que sugere que o número de nós da rede deve estar na faixa entre $1 \%$ e $10 \%$ do número de artigos da busca.

Considerando-se que as palavras chaves têm relação direta com os assuntos abordados nos artigos, esta rede permitiu identificar áreas temáticas que mais se relacionaram com os temas "redes sociais" e "cooperação". As cores e espessuras representaram a intensidade das relações entre os nós. A pesquisa identificou seis áreas temáticas principais:

- Desempenho: relacionada nos textos com o atingimento das metas esperadas nas relações de parcerias das redes. Verifica a existência ou não cooperação e benefícios advindos da interação entre os atores.

- Evolução: com foco em como estas redes se comportam ao longo do tempo, analisa a frequência de entrada e saída dos atores, o comportamento dos mesmos e a intensidade das relações.

- Gestão: aparece nos textos com foco em como estas redes podem ser mais bem geridas e administradas, melhorando os seus resultados.

- Gráficos: a maioria dos trabalhos que abordou esta área focou em "tirar uma foto" do modelo da rede existente, ou seja, analisar as relações e os resultados, sem se preocupar em criar um novo modelo.

- Modelo: surge aqui uma preocupação em mapear e modelar redes da forma a obter os melhores resultados.

- Teoria dos jogos: representada mais especificamente pelo jogo "dilema do prisioneiro", aparece nos textos com o interesse em estimular a cooperação e analisar a interação entre as pessoas. O dilema do prisioneiro é um jogo que estimula a cooperação entre os participantes pune a falta dela. Através dele é possível identificar padrões de comportamento. 
Figura 2 - Rede de palavras chaves que apareceram mais do que seis vezes.

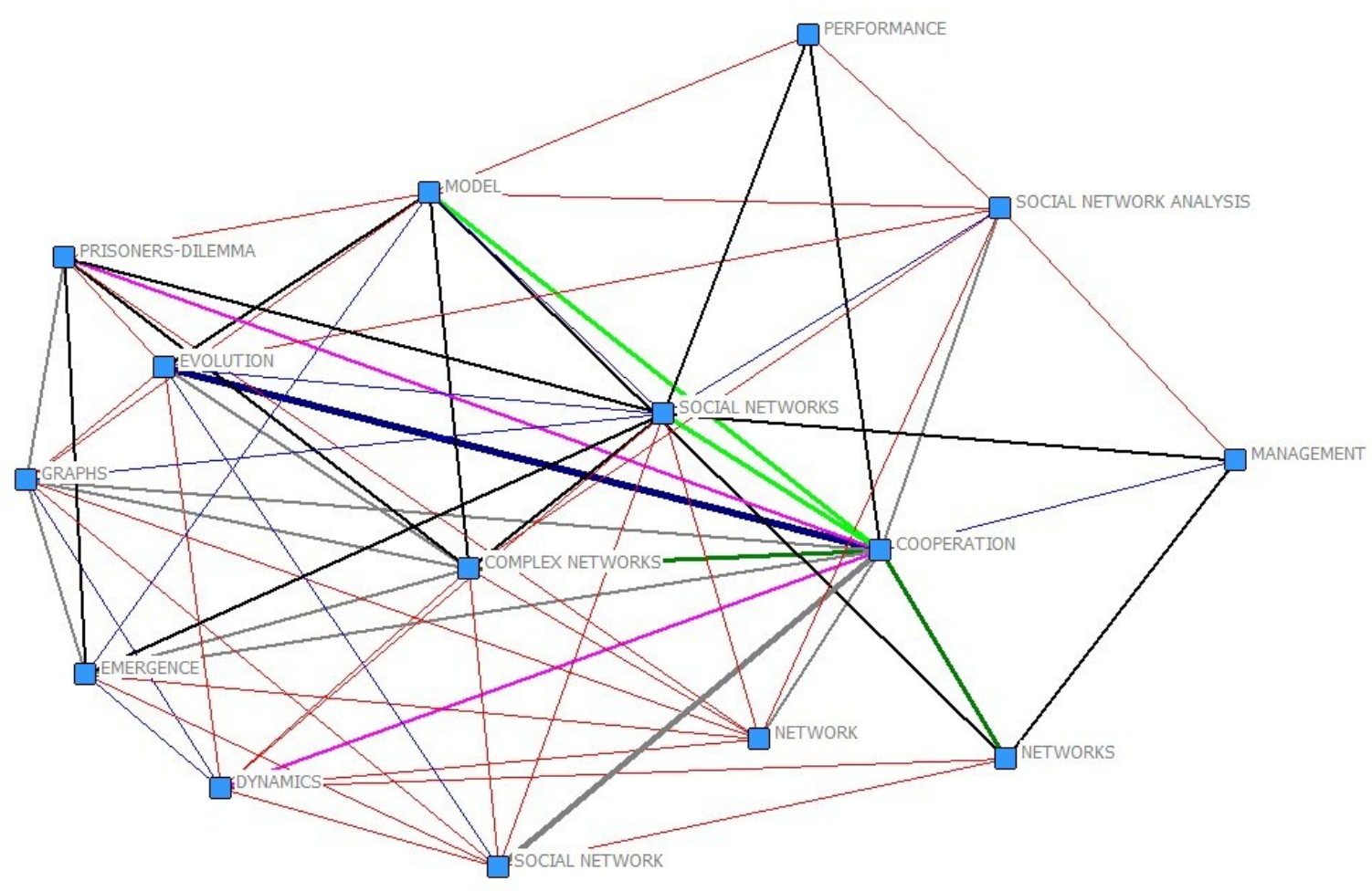

A Tabela 1 mostra a relação de publicações por área e ano, onde é possível verificar que até 2004, as publicações apareceram de forma discreta. De 2005 em diante o interesse pelo tema aumentou a as publicações cresceram de forma significativa.

Tabela 1 - Publicações por área e ano.

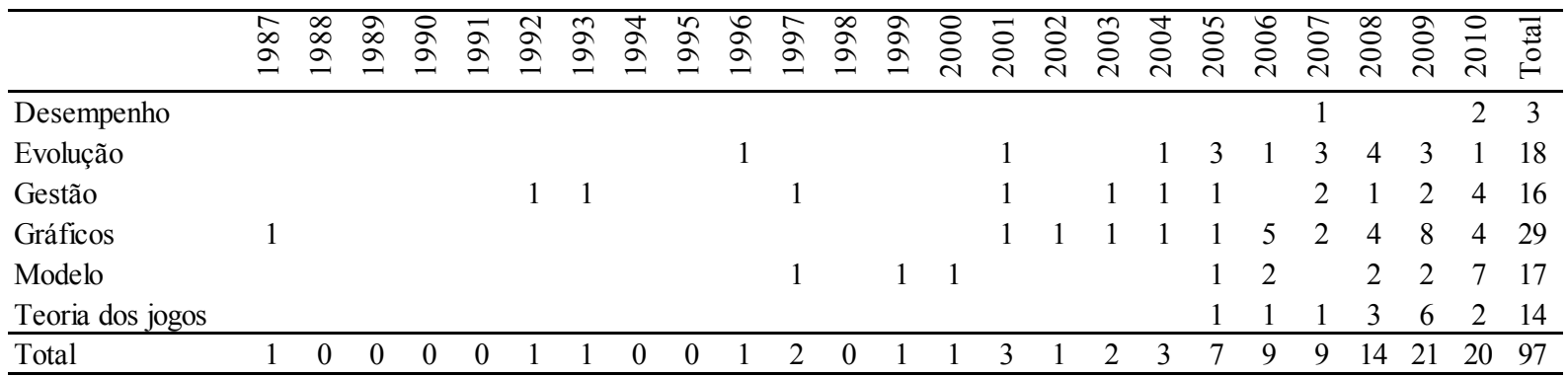

A Figura 3 apresenta graficamente a distribuição das áreas, entre os anos de 2005 e 2010, onde houve maior concentração de publicações. Até 2005 existia basicamente a preocupação de identificação de novas formas de gestão, diante da crescente pressão por inovação e dos tempos cada vez menores para o 
desenvolvimento de novos produtos. A partir de 2005, a área mais presente foi gráficos, representando o esquema de interação entre os atores.

Figura 3 - Distribuição das áreas entre 2005 e 2010.

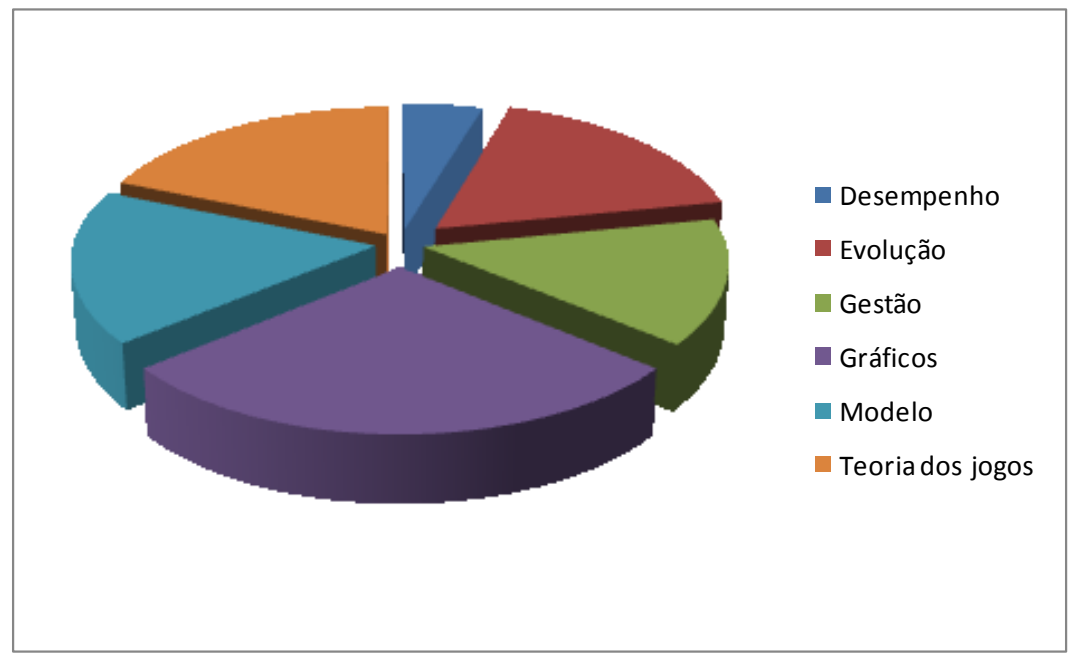

Na sequência, vemos as áreas: evolução, que foca no desenvolvimento das empresas ao longo do tempo; modelo, que indica a necessidade da construção de esquema definido de trabalho; gestão, diretamente ligada à estratégia das empresas; teoria dos jogos, no contexto do incentivo à cooperação; desempenho, representando o interesse em obtenção de vantagem competitiva.

Após a análise das publicações, foram realizadas as análises de citações. A primeira delas foi a análise dos 19 artigos com mais de vinte citações (Ver Tabela 2). 
Tabela 2 - Artigos com mais de dez citações.

\begin{tabular}{llc}
\hline Artigo & Periódico & Citações \\
\hline Walker et al. (1997) & Organization Science & 265 \\
Tsai (2002) & Organization Science & 125 \\
Giuliani and Bell (2005) & Research Police & 66 \\
Eguiluz e Zimmermann (2005) & American Journal of Sociology & 65 \\
Wagner and Leydesdorff (2005) & Research Police & 51 \\
Costenbader and Valente (2003) & Social Networks & 49 \\
Iacobucci and Hopkins (1992) & Journal of Marketing Research & 46 \\
Chwe (1999) & American Journal of Sociology & 44 \\
Daveni and Kesner (1993) & Organization Science & 41 \\
Giuliani (2007) & Journal of Economic Geography & 39 \\
Croft et al. (2006) & Behavioral Ecology and Sociobiology & 34 \\
Poncela et al. (2008) & PLoS One & 28 \\
Brandt and Sigmund (2005) & Proceedings of the National Academy of & 27 \\
Flap and Volker (2001) & Social Networks & 26 \\
Cantner and Graf(2006) & Research Police & 25 \\
Wuyts et al. (2004) & Journal of Marketing Research & 24 \\
Ferrin et al. (2006) & Journal of Applied Psychology & 23 \\
McDonald (2007) & Proceedings of the National Academy of & 23 \\
Hutt et al(2000) & Sloan Management Review & 21 \\
\hline
\end{tabular}

\section{A Figura 4 apresenta graficamente a evolução destas citações ao longo do} tempo.

Figura 4 - Evolução das citações dos 19 trabalhos mais citados.

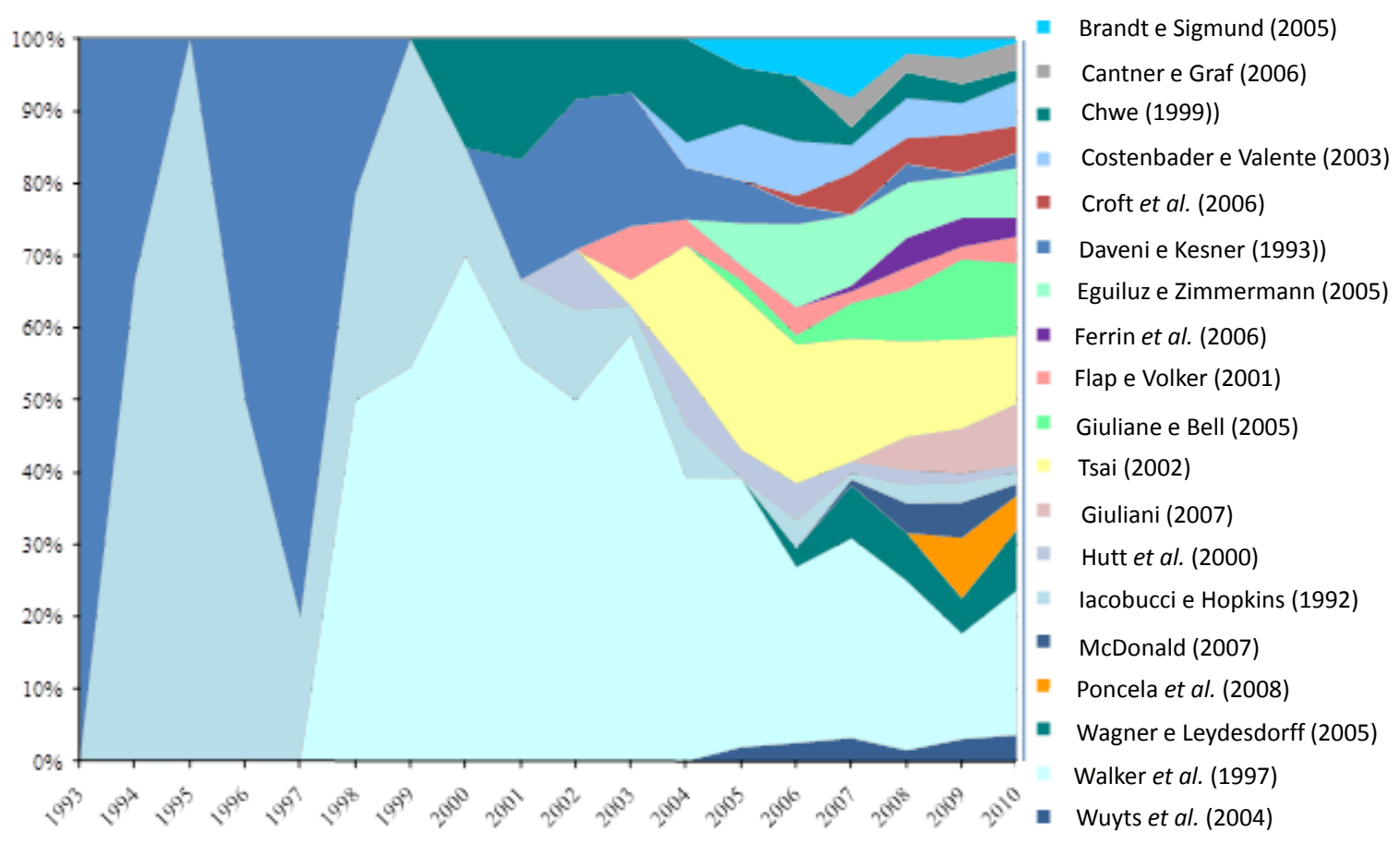

Revista Produção Online, Florianópolis, SC, v.13, n. 2, p. 634-654, abr./jun. 2013. 
De 1993 a 1999 vemos que receberam citações apenas os trabalhos Daveni e Kesner (1993), o qual analisou o impacto das aquisições nas redes sociais; lacobucci e Hopkins (1992), que focou na modelagem das redes; Walker et al. (1997), que abordou a importância do capital social na formação de redes de indústrias.

De 2000 a 2005, mais onze trabalhos passaram a receber citações, sendo eles Brandt e Sigmund (2005), que analisou a reciprocidade ao longo do tempo; Chwe (1999), que estudou as estratégias usadas na formação de redes, bem como os comportamentos identificados em ações coletivas; Costenbader e Valente (2003), que trabalhou a medição de centralidade das redes; Eguiluz e Zimmermann (2005), que identificou a importância dos líderes como um potencial diferencial; Flap e Volker (2001), que verificou o papel do capital social em diferentes tipos de redes; Giuliani e Bell (2005), o qual analisou o desempenho de empresas concentradas em clusters, com relação aos aspectos de inovação e aprendizado; Hutt et al. (2000), que realizou um estudo de caso para explorar a arquitetura de alianças estratégicas e identificar padrões de comunicação entre os participantes; Tsai (2002), o qual investigou a eficácia dos mecanismos de coordenação em matéria de partilha de conhecimentos em redes intra-organizacionais; Wagner e Leydesdorff (2005), que analisou a cooperação internacional através de co-autoria de trabalhos científicos; Wuyts et al. (2004), que identificou a intensidade das interações como um fator importante.

Num período mais recente, de 2006 a 2010, os demais trabalhos passaram a ser citados. Dentre eles Cantner e Graf (2006), que estudou uma rede social na Itália e analisou os resultados quanto à inovação; Croft et al. (2006), que investigou a cooperação natural numa população de animais; Ferrin et al. (2006), o qual pesquisou os efeitos diretos e indiretos da existência de confiança nas relações de cooperação; Giuliani (2007), que verificou se a proximidade entre as empresas numa rede de cooperação favorece ou não a inovação; McDonald (2007), o qual analisou a importância da conectividade num grupo de animais; Poncela et al. (2008), o qual identificou a capacidade de atração de ligação de um determinado nó.

A segunda análise de citações foi a análise da rede de co-citação de artigos, que indica artigos que foram citados conjuntamente nos trabalhos (ver Figura 5). 
Nesta rede é possível verificar que existem trabalhos bem centrais, tais como Eguiluz e Zimmermann (2005), Hu et al. (2007), Vega-Redondo (2006), entre outros.

Figura 5 - Rede de co-citação.

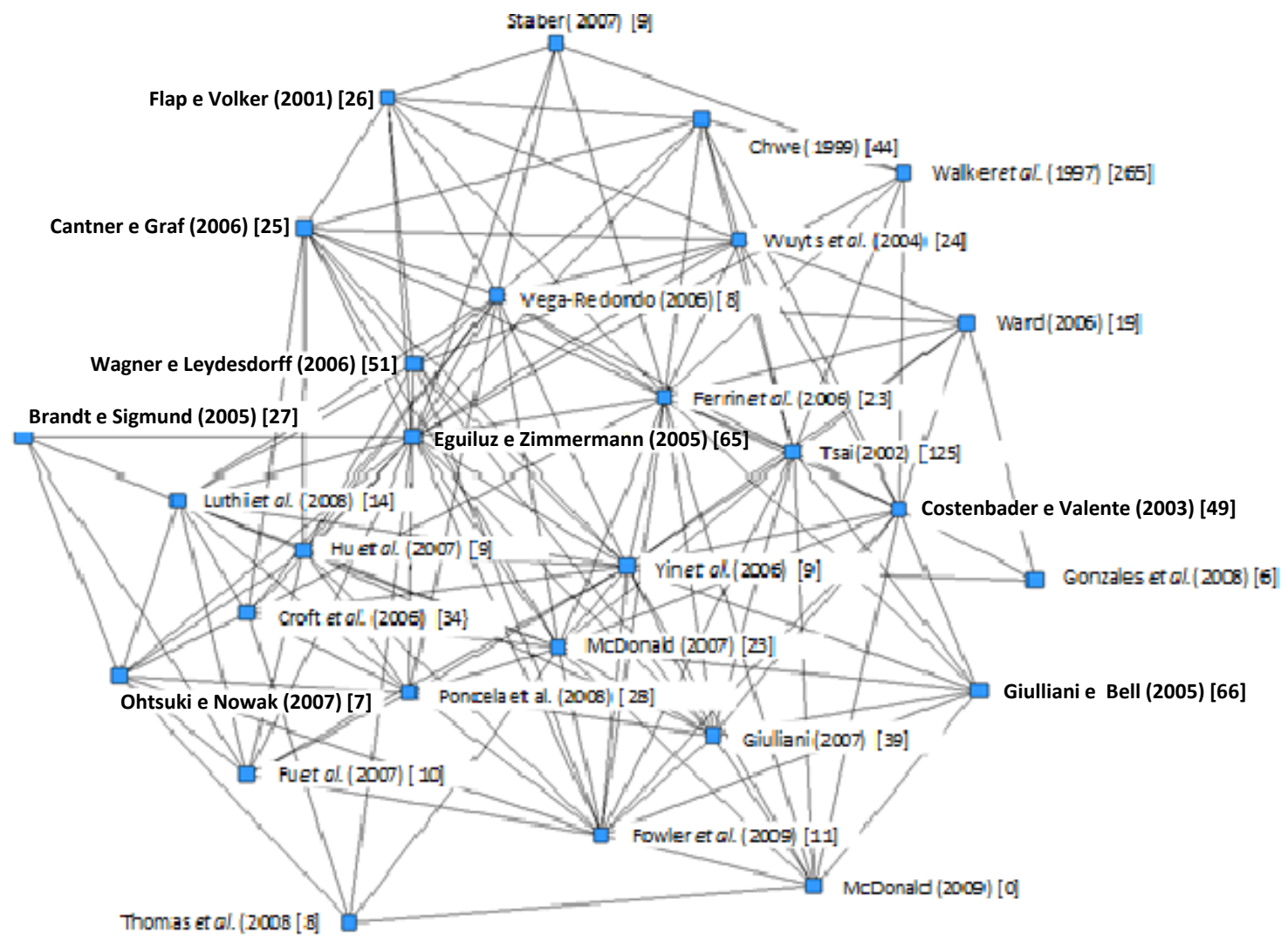

Outra rede analisada foi a rede de citação cruzada, que apresenta os autores que citam uns aos outros nos trabalhos (ver Figura 6). As informações entre parênteses representam os anos e entre colchetes, o número de citações recebidas pelos trabalhos.

Esta rede permite identificar possíveis clusters de autores que podem indicar correntes de pesquisa. Foram identificados oito agrupamentos de autores tratando do mesmo tema, sendo os temas: confiança, alianças estratégicas, teoria dos jogos, capital social, intensidade de interação, cooperação natural, reciprocidade e inovação. 
Figura 6 - Rede de citação cruzada.

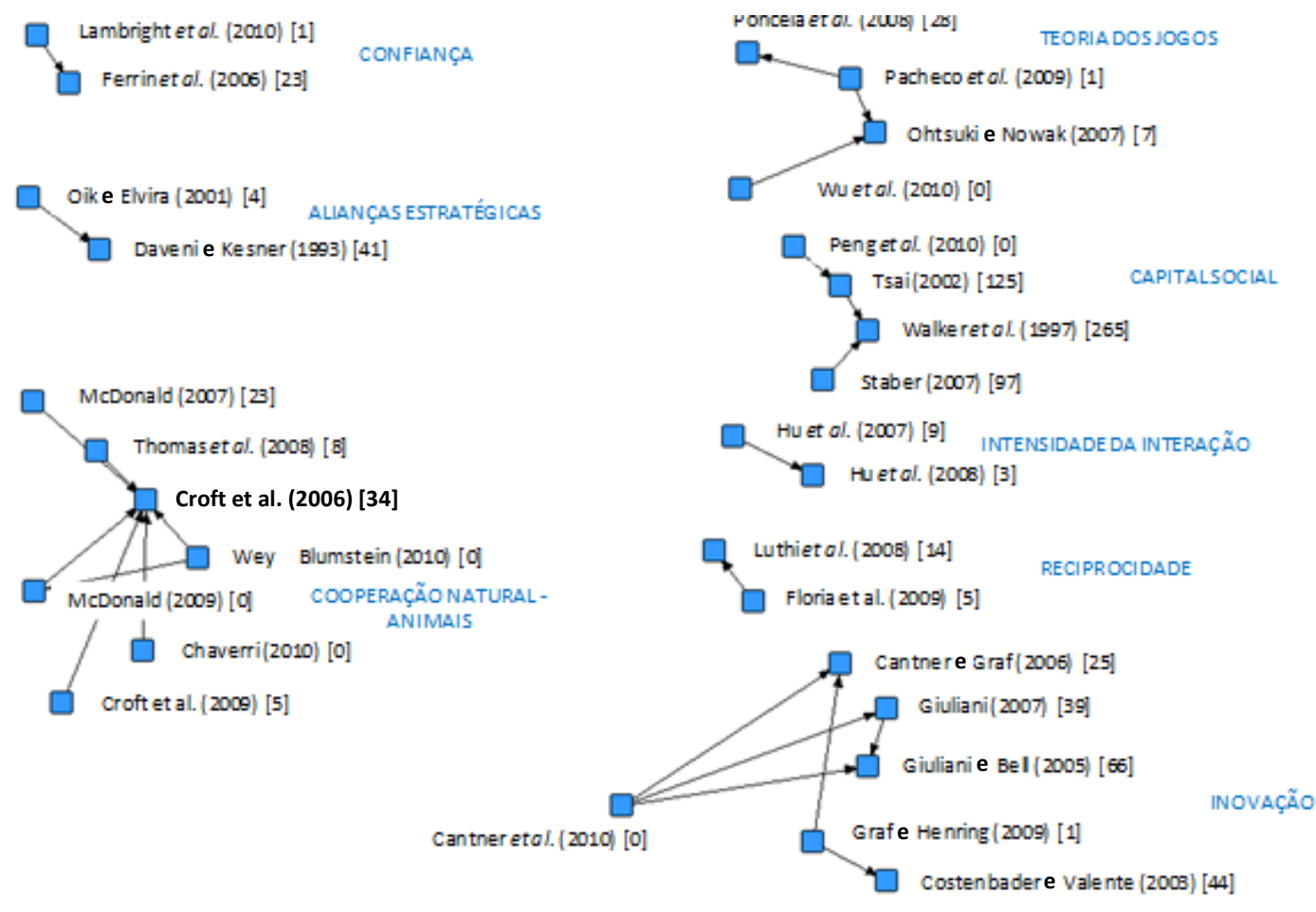

Analisando a similaridade de temas abordados, surgiram Lambright et al. (2010) e Ferrin et al. (2006), que se preocuparam com a importância da confiança nas redes sociais. Olk e Elvira (2001) e Daveni e Kesner (1993) viram as redes como uma forma estratégica de se relacionar. Já Poncela et al. (2008), Pacheco et al. (2209), Ohtsuki e Nowak (2007) e Wu et al. (2010) usaram a teoria dos jogos para entenderem as interações entre as pessoas e possíveis padrões de comportamento. Num outro grupo, Peng et al. (2010), Tsai (2002), Walker et al. (1997) e Staber (2007) consideraram importante entender a importância do capital social nas redes. Hu et al. (2007) e Hu et al. (2008) mostraram que a intensidade das relações pode influenciar o comportamento das redes e os resultados obtidos. De forma similar ao último grupo de trabalhos, Luthi et al. (2008) e Floria et al. (2009) estudaram a reciprocidade entre os atores.

Um grupo que está pouco relacionado a esta pesquisa, mas que apareceu de forma significante na amostra foi o grupo que estudou a relação entre animais, 
formado por McDonald (2007), Thomas et al. (2008), Croft et al. (2006), Wey e Blumstein (2010), McDonald (2009), Chaverri (2010) e Croft et al. (2009).

Por último, um grupo preocupado com a vertente da inovação, composto pelos trabalhos Cantner e Graf (2006), Giuliani (2007), Giuliani e Bell (2005), Graf e Henring (2009), Costenbader e Valente (2003) e Cantner et al. (2010).

Por fim, foi analisada a rede de colaboração entre países no desenvolvimento das pesquisas dos 97 artigos (ver Figura 7). No canto esquerdo superior apareceram os países que realizaram os trabalhos de forma isolada, ou seja, sem se relacionar com nenhum outro país, como é o caso do Brasil, Áustria, Hungria, Nigéria e Venezuela. Os Estados unidos trabalharam em parceria com Israel, Singapura, Holanda e Coréia do Sul, enquanto a Inglaterra apareceu de forma bem central numa rede maior, a qual envolveu vinte países.

Figura 7 - Rede de colaboração entre países.

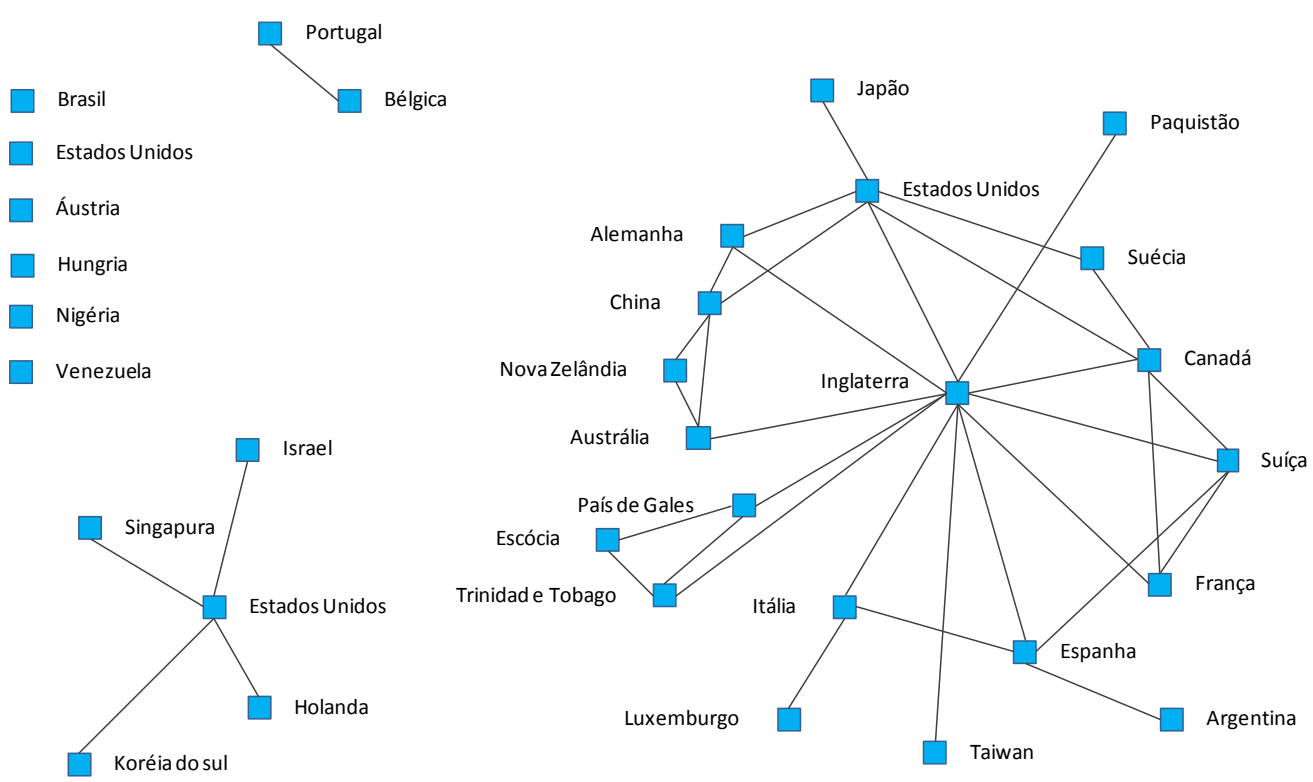

\section{CONCLUSÕES}

Esta pesquisa permitiu analisar quantitativamente e qualitativamente a teoria de redes sociais e cooperação. Apesar de não se tratar de um tema novo, a literatura evoluiu de forma significativa principalmente nos últimos cinco anos. Sua maior relevância é o fato de que cada vez mais é preciso estar conectado em rede 
para obter vantagens tais como desenvolver e compartilhar competências; diminuir custos e riscos de pesquisa / produção; compartilhar e gerar novos conhecimentos; ter acesso a novos mercados, entre outras.

Este tema interessa à comunidade acadêmica por se tratar de um assunto que a própria academia precisou conhecer e até mesmo adotar como prática de pesquisa. As barreiras de acesso ao conhecimento praticamente desapareceram com o advento das redes sociais e os autores, bem como as universidades, passaram a estudar e produzir trabalhos acadêmicos organizados em rede e cooperando.

Por meio de uma varredura horizontal foi possível verificar no estudo bibliométrico, que os periódicos que mais publicaram foram: Behavioral Ecology and Sociobiology, Organization Science, Physica A-Statistical Mechanics and its Applications, Proceedings of the National Academy of Sciences of the USA, Research Policy.

A análise das áreas demonstrou a necessidade de empresas buscarem novas formas de gestão de seus negócios, com foco na cooperação por meio de redes sociais, em busca de melhores resultados e obtenção de vantagem competitiva. A análise das redes de citações mostrou que não existe concentração de trabalhos em autores específicos, o que confirma a multidisciplinaridade do tema.

\section{Agradecimentos}

Os autores agradecem os comentários dos revisores, que muito contribuíram com o texto.

\section{REFERÊNCIAS}

AMATO NETO, J. Redes de cooperação produtiva e clusters regionais: oportunidades para as pequenas e médias empresas. São Paulo: Atlas, 2000.

AMATO NETO, J. Redecoop: núcleo de pesquisa em redes de cooperação e gestão do conhecimento. Revista Produção Online, v. 6, n. 3, p. 1-26, 2006.

BARNES, JA. Class and committees in a Norwegian Island Parish. Human relations, v. 7, n. 1, p. 39-58, 1954. 
BORGATTI, S.; EVERETT, M.; FREEMAN, L. Ucinet for windows: software for social network analysis. Analytic Technologies, 2002.

BRANDT, H.; SIGMUND, K. Indirect reciprocity, image scoring, and moral hazard. Proceedings of the National Academy of Sciences of the United States of America, v. 102, n. 7, p. 2666-2670, 2005.

CALDAS, M.P. "Contribuição teórica": como assim cara pálida. Fórum desenvolvimento de teoria, 2003.

CANDIDO, G.A.; VIEIRA, L.M.M. Aplicação dos conceitos de redes interorganizacionais no setor varejista: uma proposta de aplicação dos conceitos de brokers e operadores logísticos em empresas distribuidoras de alimentos. Revista Produção Online, v. 6, n. 2, p. 1-23, 2006.

CANTNER, U.; GRAF, H. The network of innovators in Jena: an application of social network analysis. Research Policy, v. 35, n. 4, p. 463-480, 2006.

CANTNER, U.; MEDER, A.; WAL, A.L.J. Innovator networks and regional knowledge base. Technovation, v. 30, n. 9-10, p. 496-507, 2010.

CARDOSO, J.F.; CARDOSO, J.G.; CASAROTTO FILHO, N. A estrutura intelectual de investigação de aglomerações produtivas: um estudo no âmbito da Abepro.

Revista Produção Online, v. 11, n. 3, p. 871-890, 2011.

CARVALHO, M. M.. Inovação: estratégias e comunidades de conhecimento. São Paulo: Atlas, 2009.

CHAVERRI, G. Comparative social network analysis in a leaf-roosting bat.

Behavioral Ecology and Sociobiology, v. 64, n. 10, p. 1619-1630, 2010.

CHESNAIS, F. A mundialização do capital. São Paulo: Xamã, 1996.

CHWE, M.S.Y. Structure and strategy in collective action. American Journal of Sociology, v. 105, n. 1, p. 128-156, 1999.

COSTENBADER, E.; VALENTE, T.W. The stability of centrality measures when networks are sampled. Social Networks, v. 25, n. 4, p. 283-307, 2003.

CROFT, D.P.; JAMES, R.; THOMAS, P.O.R. Social structure and co-operative interactions in a wild population of guppies. Behavioral Ecology and Sociobiology, v. 59, n. 5, p. 644-650, 2006.

CROFT, D.P.; KRAUSE, J.; DARDEN, S.K.; RAMNARINE, IW; FARIA, JJ; JAMES, R Behavioral trait assortment in a social network: patterns and implications. Behavioral Ecology and Sociobiology, v. 63, n. 10, p. 1495-1503, 2009. 
DAVENI, R.A.; KESNER, I.F. Top managerial prestige, power and tender offer response - a study case of elite social networks and target firm cooperation during takeovers. Organization Science, v. 4, n. 2, p. 123-151, 1993.

DU, WB; CAO, XB; ZHENG, HR; ZHOU, H. Evolutionary games in multi-agent systems of weighted social networks. International Journal of modern Physics, $\mathrm{v}$. 20, n. 5, p. 701-710, 2010.

EGUILUZ, V.M.; ZIMMERMANN, M.G. Cooperation and the emergence of role differentiation in the dynamics of social networks. American Journal of Sociology, v. 110 , n. 4, p. 977-1008, 2005.

FERRIN, D.L.; DIRKS, K.T.; SHAH, P.P. Direct and indirect effects of third-party relationships on interpersonal trust. Journal of Applied Psychology, v. 91, n. 4, p. 870-883, 2006.

FLAP, H. VOLKER. B. Goal specific social capital and job satisfaction: effects of different types of networks on instrumental and social aspects of work. Social Network, v. 23, n. 4, p. 297-320, 2001.

FLORIA, L.M.; GRACIA-LAZARO, C.; GOMEZ-GARDENES, J.; MORENO Y. Social network reciprocity as a phase transition in evolutionary cooperation. Physical Review E, v. 79, n. 2, 2009.

FORESTI, N. Estudo da contribuição das revistas brasileiras de biblioteconomia e ciência da informação enquanto fonte de referência para pesquisa. Dissertação (Mestrado) - Departamento de Biblioteconomia da Universidade de Brasília, 1989.

FOWLER, JH; DAWES, CT; CHRISTAKIS, NA. Model of genetic variation in human social networks. Proceedings of the National Academy of Sciences of the United States of America, v. 106, n. 6, 2009.

FU, F; CHEN, XJ; LIU, LH; WANG, L. Social dilemmas in an online social network: The structure and evolution of cooperation. Physics Letters, v. 371, n. 1-2, p. 58-64, 2007.

GIULANI, E. The selective nature of knowledge networks in clusters: evidence from the wine industry. Journal of Economic Geography, v. 7, n. 2, p. 139-168, 2007.

GIULIANI, E.; BELL, M. The micro-determinants of meso-level learning and innovation: evidence from a Chilean wine cluster. Research Policy, v. 34, n. 1, p. 4768, 2005.

GRAF, H.; HENNING, T. public research in regional networks of innovators: a comparative study of four east German regions. Regional Studies, v. 43, n. 10, p. 1349-1368, 2009. 
HARABI, N. The impact of vertical R\&D cooperation on firm innovation: an empirical investigation. Economics of innovation and new technology, v. 11, n. 2, p. 93108, 2002.

HU, M.B.; JIANG, R.; WU, Q.S.; WU YH. Simulating the wealth distribution with a richest-following strategy on scale-free network. Physical A-Statistical Mechanics and its Applications, v. 381, p. 467-472, 2007.

HU, M.B.; JIANG, R.; WU, YH; WANG, RL; WU, QS. Properties of wealth distribution in multi-agent systems of a complex network. Physical A-Statistical Mechanics and its Applications, v. 387, n. 23, p. 5862-5867, 2008.

HUTT, M.D.; STAFFORD, E.R.; WALKER, B.A.; REINGEN, PH. Case study defining the social network of a strategic alliance. Sloan Management Review, $v$. 41 , n. 2, p. $51,2000$.

IACOBUCCI, D.; HOPKINS, N. Modeling dyadic interactions and networks in marketing. Journal of Marketing Research, v. 29, n. 1, p. 5-17, 1992.

IKPAAHINDI, L. An Overview of bibliometrics: its measurements, laws and their applications. Libri, v. 35, n. 2, p.163-176, 1985.

KEMCZINSKI, A.; MAREK, J,; HOUSELL, M.S.; GASPARINI, I. Colaboração e cooperação: pertinência, concorrência ou complementaridade. Revista Produção Online, v. 7, n. 3, p. 1-15, 2007.

LAMBRIGHT, K.T.; MISCHEN, P.A.; LARAMEE, C.B. Building trust in public and nonprofit networks personal, dyadic, and third-party influences. American Review of Public Administration, v. 40, n. 1, p. 64-82, 2010.

LUTHI, L.; PESTELACCI, E.; TOMASSINI, M. Cooperation and community structure in social networks. Physical A-Statistical Mechanics and its Applications, v. 387, n. 4, p. 955-966, 2008.

MCDONALD, D.B. Predicting fate from early connectivity in a social network. Proceedings of the National Academy of Sciences of the United States of America, v. 104, n. 26, p. 10910-10914, 2007.

MCDONALD, D.B. Young-boy networks without kin clusters in a lek-mating manakin. Behavioral Ecology and Sociobiology, v. 63, n. 7, p. 1029-1034, 2009.

NEELY, A. The evolution of performance measurement research: developments in the last decade and a research agenda for the next. International Journal of Operations \& Production Management, v. 25, n. 12, p. 1264-1277, 2005.

OHTSUKI, H.; NOWAK, M.A. Direct reciprocity on graphs. Journal of Theoretical Biology, v. 247, n. 3, p. 462-470, 2007. 
OLK, P.; ELVIRA, M. Friends and strategic agents - the role of friendship and discretion in negotiating strategic alliances. Group Organization Management, v. 26, n. 2, p. 124-164, 2001.

OPRIME, P.C.; TOLEDO J.C.; TRISTÃO, H.M.; PIMENTA, M.L. Análise dos relacionamentos e cooperação entre empresas do cluster industrial de jóias e folhados de Limeira. Revista Produção Online, v. 9, n. 4, p. 1-15, 2009.

PACHECO, J.M.; PINHEIRO, F.L.; SANTOS, F.C. population structure induces a symmetry breaking favoring the emergence of cooperation. Plos Computational Biology, v. 5, n. 12, 2009.

PENG, T.J.A.; LIN, N.J.; MARTINEZ, V.; YU, CMJ. Managing triads in a military avionics service maintenance network in Taiwan. International Journal of Operations \& Production Management, v. 30, n. 3-4, p. 398-422, 2010.

PONCELA, J.; GOMEZ-GARDENES, J.; FLORIA, L.M. et al. Complex cooperative networks from evolutionary preferential attachment. Plos One, v. 3, n. 6, p. 2449, 2008.

PRASAD, S. TATA, J. Publications patterns concerning the role of teams/groups in the information systems literature from 1990 to 1999. Information \& Management, v. 42, n.8, p. 1137-1148, 2005.

RIVELLINI, G; RIZZI, E; ZACCARIN, S. The science network in Italian population research: An analysis according to the social network perspective. Scientometrics, v. 67, n. 3 , p. $407-418,2006$.

SCHILDT, H.A. Sitkis: Software for bibliometric data management and analysis. Helsinki. Institute of Strategy and International Business, v. 6.1, 2002.

SCHMITZ, H. Small firms and flexible specialization in LCD's. Institute of Development Studies, 1989.

STABER, U. Contextualizing research on social capital in regional clusters. International Journal of Urban and Regional Research, v. 31, p. 505-521, 2007.

TALAMO, J.R.; CARVALHO, M.M. Redes de cooperação com foco em inovação: um estudo exploratório. Gestão \& Produção, v. 17, n. 4, p. 747-760, 2010a.

TALAMO, J.R.; CARVALHO, M.M. Seleção dos objetivos fundamentais de uma rede de cooperação empresarial. Gestão \& Produção, v. 11, n. 2, p. 239-250, 2010b.

THOMAS, P.O.R; CROFT, D.P.; MORRELL, L.J.; DAVIS A.; FARIA, JJ; DYER, JRG; PIYAPONG, C.; RAMNARINE, I; RUXTON, GD. Does defection during predator inspection affect social structure in wild shoals of guppies? Animal Behaviour, v. 75, p. 43-53, 2008. 
TSAI, W.P. Social structure of "coopetition" within a multiunit organization: Coordination, competition, and intraorganizational knowledge sharing. Organization Science, v. 13, n. 2, p. 179-190, 2002.

VEGA-REDONDO, F. Building up social capital in a changing world. Journal of Economic Dynamics \& Control, v. 30, n. 11, p. 2305-2338, 2006.

WAGNER, C.S.; LEYDESDORFF, L. Network structure, self-organization, and the growth of international collaboration in science. Research Policy, v. 34, n. 10, p. 1608-1618, 2005.

WALKER, G.; KOGUT, B.; SHAN, W.J. Social capital, structural holes and the formation of an industry network. Organization Science, v. 8, n. 2, p. 109-125, 1997.

WARD, $\mathrm{H}$. International linkages and environmental sustainability: The effectiveness of the regime network. Journal of Peace Research, v. 43, n. 2, p. 149-166, 2006.

WASSERMAN, S.; FAUST, K. Social network analysis: methods and applications. Cambridge University Press, 1994.

WEY, T.W.; BLUMSTEIRN, D.T. Social cohesion in yellow-bellied marmots is established through age and kin structuring. Animal Behaviour, v. 79, n. 6, p. 13461352, 2010.

WILLIAMSON, OE. Markets, hierarquies and the modern corporation: an unfolding perspective. Journal of Economic Behavior and organization, v. 17, p. 335-352, 1992.

WU, B.; ZHOU, D.; FU, F.; LUO, QJ; WANG, L; TRAULSEN, A. Evolution of cooperation on stochastic dynamical networks. Plos One, v. 5, n. 6, 2010.

WUYTS, S.; STREMERSCH, S. VAN DEN BULTE, C.; FRANSES, PH. Vertical marketing systems for complex products: A triadic perspective. Journal of Marketing Research, v. 41, n. 4, p. 479-487, 2004.

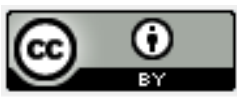

Artigo recebido em 10/02/2012 e aceito para publicação em 17/04/2013. 
Anexo 1 - Tipologia de redes.

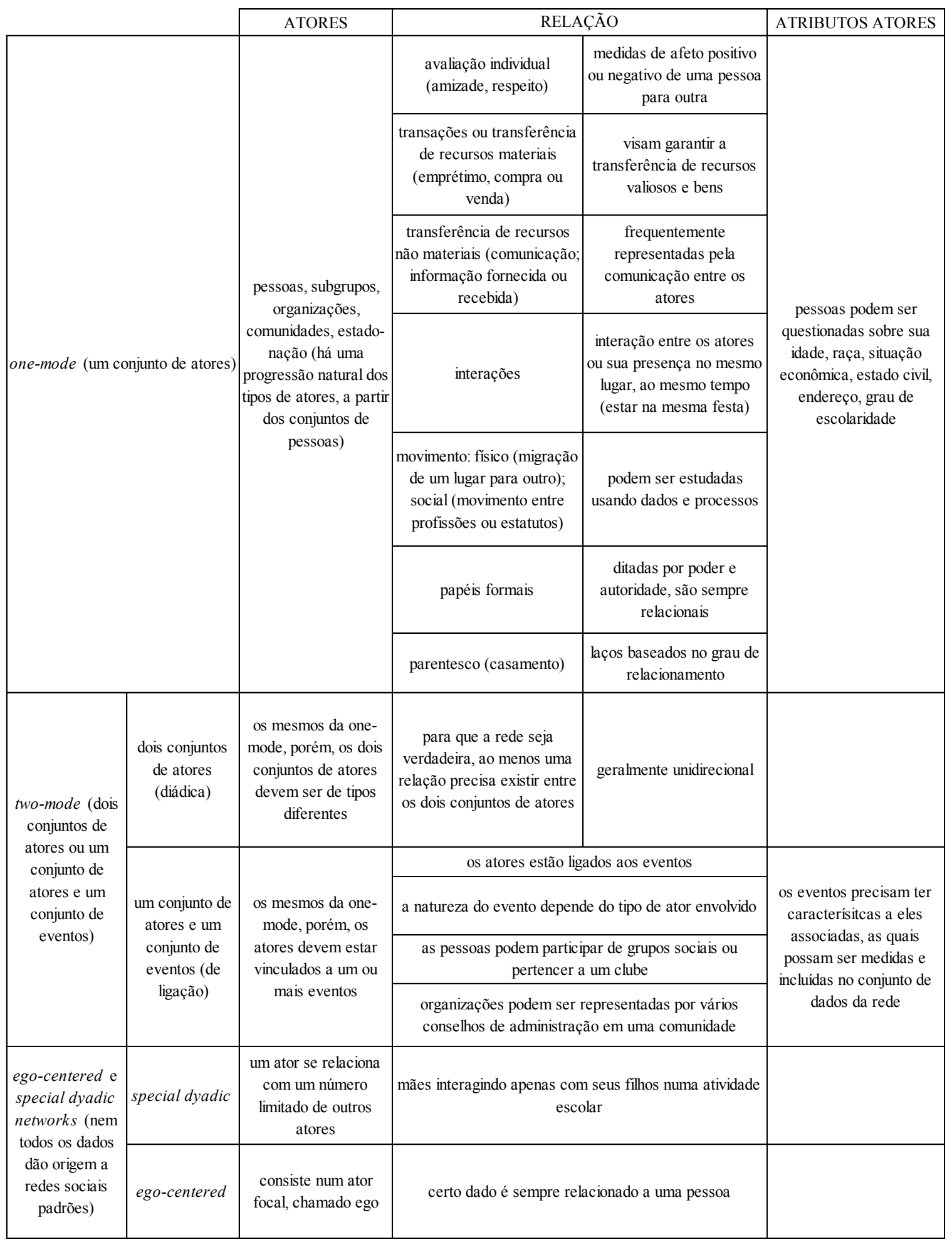

Fonte - Adaptado de Wasserman e Faust (1994).

Revista Produção Online, Florianópolis, SC, v.13, n. 2, p. 634-654, abr./jun. 2013. 
Anexo 2 - Publicações por periódico e ano.

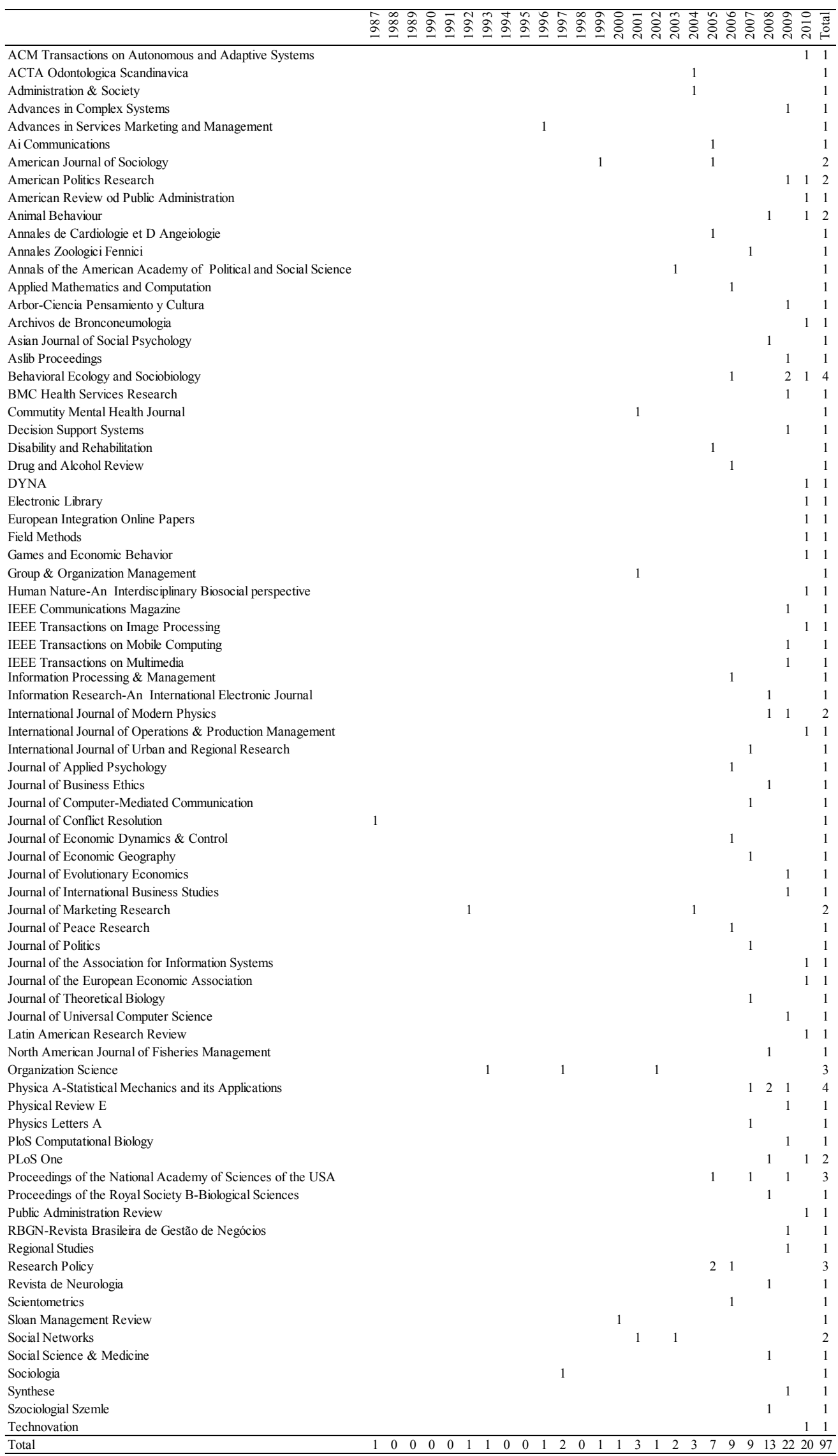

Revista Produção Online, Florianópolis, SC, v.13, n. 2, p. 634-654, abr./jun. 2013. 